\title{
Eigenverantwortung und Darmkrebsvorsorge: warum und wie, die Chronikerrichtlinie revidiert werden sollte ${ }^{1}$
}

\section{HARALD SCHMIDT}

Harald Schmidt ist z. Zt. wissenschaftlicher Mitarbeiter am Center for Health Incentives and Behavioral Economics, School of Medicine, University of Pennsylvania, USA.

Das Konzept der Eigenverantwortung ist ein schillernder Begriff, der von besonderer Relevanz für die Debatte um die Chronikerrichtline ist. Die Anwendung der Richtlinie wird hier für den Fall der Darmkrebsvorsorge nach dem Ansatz der Verfahrensgerechtigkeit diskutiert. Neben der konzeptuellen Analyse werden neue Umfragedaten und Ergebnisse halb-strukturierter qualitativer Interviews mit Ärzten vorgestellt, die in der Zusammenschau nahelegen, dass die Chronikerrichtline stark revisionsbedürftig ist. Abschließend werden daher konkrete Änderungsvorschläge entwickelt.

\section{Einleitung}

Das Konzept der Eigenverantwortung spielt eine prominente Rolle in den Bestimmungen des fünften Buches des Sozialgesetzbuches (SGB V). Seine genaue Bedeutung ist allerdings schillernd und alles andere als eindeutig (Buyx 2005; Schmidt 2007a; Schmidt 2007b; Höfling 2009; Huster 2012; Marckmann 2005). An anderer Stelle habe ich unterschiedliche Bedeutungen in prospektiver und retrospektiver Verwendung des Begriffs unterschieden und argumentiert, dass ein Teil der Verwirrung in der akademischen und gesundheitspolitischen Diskussion daher rührt, dass er unausweichlich zwischen deskriptivem und normativem Gehalt oszilliert (Schmidt 2009; Schmidt 2012). Die Diskussion ist weiterhin dadurch erschwert, dass es eine Reihe verschiedener normativer Theorien gibt, die zu prinzipiell unterschiedli- chen Anwendungen von Begriffen der Eigenverantwortung führen können. Ein Problem bei der Umsetzung dieser Theorien ist zudem, dass in pluralistischen Gesellschaften generell nicht ein einziger Ansatz universelle Zustimmung findet, was zu Legitimitätsproblemen bei der

\footnotetext{
1 Eine frühere Fassung dieses Beitrags wurde auf dem Workshop »Public Health EthicsScientific methods, foundational concepts and case analyses « an der Medizinischen Hochschule Hannover im Februar 2011 vorgestellt. Ich danke den Diskussionsteilnehmern wie auch Bettina Schöne-Seiffert und Johann Ach für konstruktives Feedback, und insbesondere Stephen John für kritische Durchsicht des Manuskriptes, das in einem englischsprachigen Sammelband der Tagungsbeiträge erscheinen wird. Der Text hier folgt jenem Beitrag, unterscheidet sich jedoch darin, dass er erstmalig Resultate qualitativer Interviews und Umfrageergebnisse zum Thema vorstellt. Dirk Horenkamp-Sonntag und Jürgen Wasem danke ich für Rat zu spezifischen Regelungsfragen, wie weiter unten ausgeführt, und Dorothea Schmidt für kritische Durchsicht.
} 
Durchsetzung einer bestimmten Theorie führen kann. Weiterhin ist keiner der etablierten normativen Ansätze feinkörnig genug, um direkt handlungsleitend zu wirken, oder bei der Bewertung von Regelungen unmittelbar hilfreich zu sein (Schmidt 2008).

In einer solchen Situation gewinnt Verfahrensgerechtigkeit an Bedeutung (Daniels 2007). Hierbei geht es darum anzuerkennen, dass vollständige Übereinstimmung hinsichtlich leitender ethischer Normen oft schwer erreichbar ist, wenngleich Übereinstimmung bezüglich des Verfahrens möglich ist, mit dem vernünftige Menschen mit verschiedenen Grundüberzeugungen vorgehen sollten, um Regelungen $\mathrm{zu}$ finden, die auf breite und wechselseite Zustimmung stoßen. James Sabin und Norman Daniels haben im Zusammenhang mit der Frage der Priorisierung vier zentrale Bedingungen identifiziert, die Regelungen erfüllen müssen (Daniels und Sabin 1999; Daniels 2000; Daniels 2007): (1) Öffentlichkeit (Entscheidungen und die entsprechenden Begründungen sollten allgemein zugänglich sein), (2) Relevanz (Regelungen sollten von vernünftigen Erklärung begleitet sein, siehe unten), (3) Einspruchsmöglichkeit und Revidierbarkeit (Mechanismen, mit denen berechtigte Einwände vorgetragen werden können, müssen bereitstehen und die Möglichkeit enthalten, Änderungen zu bewirken), und (4) Regulierung, so dass die drei vorgenannten Bedingungen in der Praxis effektiv sind. Die Relevanz-Bedingung wird in ihrer kürzesten Fassung wie folgt bestimmt: »The rationales for limit-setting decisions should aim to provide a reasonable explanation of how the organization seeks to provide value for money in meeting the varied health needs of a defined population under reasonable resource constraints. Specifically, a rationale will be reasonable < if it appeals to evidence, reasons and principles that are accepted as relevant by [fair minded] people who are disposed to finding mutually justifiable terms of cooperation. Where possible, the relevance of reasons should be vetted by stakeholders in these decisions« (Daniels 2000: 110).

An den oben zitierten Stellen habe ich versucht, die Relevanz-Bedingung mit Bezug auf Regelungen näher zu konkretisieren, die das Ziel haben, Eigenverantwortung zu fördern. Abbildung 1

\section{Abb. 1: Sieben Bereiche, in denen Rechtfertigungen für die Angemessenheit von Regelungen zur Förderung der Eigenverantwortung erforderlich sind (Schmidt 2008)}

\section{Evidenz, Begründung und Durchführbarkeit}

Was sind die prinzipiellen Begründungen und Ziele der Regelung? Wurden sie in transparenter und öffentlicher Weise bereitgestellt, idealerweise mit Kommentar-Möglichkeit für relevante Interesseneigner? Wie sicher ist es, dass die Regelung die Ziele erreichen kann (prinzipiell und unter realen Bedingungen)? Verhalten sich die erforderlichen Mittel und Kosten proportional zu den Zielen?

\section{Stärke der Eingriffe und Zwang}

Zu welchem Maß beeinträchtigt die Regelung individuelle Freiheit? Gibt es Möglichkeiten, die Ziele über weniger stark eingreifende Mittel zu erreichen? Wenn nicht, ist das Ausmaß an Intrusion angemessen, angesichts des zu erwartenden Nutzens?

\section{Zuschreibbarkeit/Handlungsmöglichkeit}

In wie weit sind Belohnungen oder Strafen direkt freiwilligen Handlungen von Personen zuzuschreiben? In Fällen, da Handlungsmöglichkeiten beschränkt sind: Können Alternative angeboten werden? Sollen Belohnungen angeboten werden, selbst wenn Personen ihr Verhalten nicht geändert haben, jedoch die Kriterien einer Regelung erfüllen?

\section{Gerechtigkeit}

Gibt es Personengruppen (beispielsweise bestimmte sozio-ökonomische, ethnische oder regionale Untergruppen) die disproportional Vor- oder Nachteile erfahren? An welchem Punkt werden Ungleichheiten zu Ungerechtigkeiten und (wie) kann diesen in Modifizierungen der Regelung begegnet werden?

\section{Solidarität}

Insofern dem Gesundheitssystem ein implizites oder explizites Prinzip der Solidarität oder Risiko-Zerstreuung zugrunde liegt: wie wirkt sich die Regelung darauf aus? Falls die Regelung derartige Prinzipien unterminiert, sind sich alle Interesseneigner dessen bewusst, und kann die Beeinträchtigung gerechtfertigt werden?

\section{Wirkung auf Dritte}

Hat die Regelung Auswirkungen auf das Verhältnis zu Dritten, wie zum Beispiel Ärzten oder Arbeitgeber? Insofern Ärzte daran beteiligt sind festzustellen, ob jemand Kriterien erfüllt hat, die eine Strafe oder Belohnung zur Folge haben: Ist ihre Beteiligung nötig und wird sie von ihnen selbst und von den Patienten akzeptiert? Zu welchen Information bezüglich der Nutzung von Anreizprogrammen sollen Arbeitgeber Zugang haben (und zu welchen nicht)?

\section{Kohärenz}

Wie verhält sich die Regelung zu andern rechtlichen, gesundheits- oder sozialpolitischen Standards, die das Ziel haben Eigenverantwortung zu fördern? Insofern es Spannungen gibt: Wie soll mit diesen umgegangen werden (da sie typischerweise in mehrere Richtungen aufgelöst werden können)?

zeigt sieben Bereiche von zentraler normativer und struktureller Bedeutung für Gesundheitssysteme, in denen die Planung, Durchführung, und Evaluation von Regelungen zur Eigenverantwortung explizit gerechtfertigt werden sollten. Im Folgenden werde ich die Bestimmungen zur Darmkrebsvorsorge nach der Chronikerrichtlinie, basierend auf $\mathbb{} 62$ SGB V, entlang dieser Matrix diskutieren, und versuchen zu zeigen, dass zentrale Elemente aus ethischer Sicht revisionsbedürftig sind. Eine mit der Regelung neu geschaffene >Pflicht zum Nachdenken $<$ ist jedoch prinzipiell $\mathrm{zu}$ verteidigen.

\section{Methoden und Limitationen}

Das Vorgehen stützt sich im Folgenden neben einer konzeptuellen Analyse der relevanten Sachverhalte und Literatur 
auch auf neue Umfragedaten und die Ergebnisse halb-strukturierter qualitativer Interviews mit Ärzten (im Interesse der Lesbarkeit beinhaltet die männliche Form hier und weiter unten die weibliche). Im Rahmen einer vergleichenden Studie wurden $20 \mathrm{ca}$. halbstündige Interviews mit je fünf Hausärzten und Onkologen in Berlin und Philadelphia zwischen März-September 2011 durchgeführt, in der Mehrheit in einem direkten Gespräch, und in sechs Fällen telefonisch (in diesem Artikel bezieht sich die Beschreibung nur auf die Berliner Ärzte). Die Auswahl war zielorientiert (purposive sampling), individuelle Einschlusskriterien waren eine mindestens fünfjährige Berufserfahrung und ein Alter zwischen 35-65 Jahren. Ausschlusskriterien waren Ärzte, die ausschließlich oder überwiegend alternative Medizin praktizierten, und Personen, die bereits zu ethischen Fragen publiziert hatten. Weitere Kriterien waren, dass etwa die Hälfte weiblich sein sollte, und dass in jeder Gruppe von fünf Ärzten zwei ihre Praxis in Gebieten haben sollten, deren Bevölkerung gemäß dem Sozialstrukturatlas überwiegend einen niedrigen sozioökonomischen Status haben, zwei einen hohen und einer einen mittleren. Dies ließ sich für die Hausärzte gut umsetzen, jedoch weniger bei den Onkologen, die - bis auf eine Ausnahme - zumeist in größeren Kliniken oder Krankenhäusern tätig waren. Ärzte wurden über das Register der Kassenärztliche Vereinigung Berlin kontaktiert, sowie über die Deutsche Krebsgesellschaft, den Verein der niedergelassenen Hämatologen, persönlich bekannte Forscher und Ärzte, und ein sich daraus ergebendes >Schneeball<-Verfahren. Insgesamt wurden auf diese Weise 22 Ärzte mit einem standardisierten Anschreiben direkt oder indirekt angefragt, von denen zehn zu einer Befragung bereit waren. Finanzielle Anreize oder Aufwandsentschädigungen wurden nicht angeboten, und die Studie wurde gemäß den Bestimmungen der ForschungsethikKommission der London School of Economics and Political Science vom Review-Verfahren ausgenommen. Der Interview-Leitfaden (erhältlich beim Autor) enthielt 14 Fragen zu verschiedenen Aspekten der Eigenverantwortung und wurde umfangreich getestet. Die Interviews wurden elektronisch aufgenommen, transkribiert, und mit einem Pro- gramm zur computer-assistierten Analyse qualitativer Daten (NVIVO Version 8.03), und mithilfe einer in drei Zyklen entwickelten Code-Liste systematisch untersucht. Alle Interviews wurden vom Autor geführt, kodiert und analysiert.

Zusätzlich wurden Online-Umfragen innerhalb der deutschen und der US-amerikanischen Bevölkerung mit einem für den Zweck abgewandelten Fragebogen durchgeführt, in dem vor allem offene Fragen durch Likert-Skalen ersetzt und weitere textliche Vereinfachungen vorgenommen wurden, die sich nach einem Pre-Test als hilfreich erwiesen (Fragebogen wiederum beim Autor erhältlich). Die Fragen wurden über die Umfrageplattform Surveymonkey gestellt, und Links zur Umfrage über den Stichprobenanbieter Usamp an registrierte Mitglieder von (opt-in) Panels versendet. Für die Stichprobe wurden Zensus-ajustierte Gruppen nach Geschlecht und Einkommen gebildet und post-Stratifizierungs-Gewichtungen vorgenommen, um eine Zensus-gemäße Altersstruktur zu erhalten. Die Daten wurden mit dem Analysepaket JMP Pro (Version 9.0.0) untersucht (hier relevante Tests: Pearson Chi-Square, Analysis of Variance/ANOVA, Wilcoxon/KruskalWallis). Die deutsche Umfrage wurde zwischen dem 26. September und 4. Oktober 2011 durchgeführt. Fünf Prozent aller derjenigen, die über Usamp per Mail eine Einladung mit dem Titel »Umfrage zu Gesundheitsthema« erhielten, folgten zur Surveymonkey-Seite, 97,3\% nahmen darauf hin an der Umfrage teil (1\% beendete die Umfrage vorzeitig). Es wurden die Antworten von 1.000 Teilnehmern analysiert. Gemäß den Bestimmungen der Forschungsethik-Kommission der University of Pennsylvania wurde die Studie vom Review-Verfahren ausgenommen.

Obwohl die Umfrage Zensus-adjustiert ist, liegt keine Zufallsstichprobe vor, und Extrapolationen von Antworten der Teilnehmer zur Bevölkerung insgesamt sind daher nicht zulässig, sondern erfordern eine Replikation. Die qualitativen Interviews mit Ärzten basieren auf einer kleinen, wenngleich für derartige Untersuchungen üblichen Zahl von Gesprächen. Quantitative Analysen sind nur sehr begrenzt sinnvoll, und können auch hier keineswegs beanspruchen, Aussagen über die allgemeine Sicht der Ärzteschaft wieder zu geben. In beiden Fällen ist der Anspruch an die Daten daher lediglich, plausible Hypothesen zu generieren und spezifische Aspekte anderer Analyseformen zu ergänzen.

\section{Die Chronikerrichtlinie}

Die revidierte Chronikerrichtlinie nach \ 62 SGB V wurde, wie weitere Anreizprogramme auch, unter dem Wettbewerbsstärkungsgesetz (GKV-WSG) 2007 eingeführt. Nach einem konkretisierenden Beschluss des Gemeinsamen Bundesausschusses (G-BA) und weiteren Ergänzungen besagt die Regelung, dass für Patienten, die Brust-, Gebärmutter- oder Darmkrebs erleiden, eine $\mathrm{Zu}$ zahlungsgrenze von $1 \%$ der jährlichen Bruttoeinnahmen zum Lebensunterhalt gilt, wenn sie zwei Bedingungen erfüllt haben. Erstens müssen sie zu einem bestimmten Zeitpunkt an einem Beratungsgespräch zu den Vor- und Nachteilen von Vorsorgeuntersuchungen zu den jeweiligen Krankheiten teilgenommen haben (die Regelung gilt für nach dem 1. April 1987 geborene weibliche und nach dem 1. April 1962 geborene männliche Versicherte). Zweitens haben sich Versicherte, die später chronisch erkranken in einem Gespräch mit ihrem Arzt »über das weitere Vorgehen in Bezug auf eine Therapie « zu verständigen, und Ärzte sind verpflichtet, eine entsprechende Bescheinigung über »therapiegerechtes « Verhalten auszustellen. Die Ausstellung darf verweigert werden, wenn »der $\mathrm{Pa}$ tient ausdrücklich erklärt, sich entgegen der gemeinsamen Verständigung « $\mathrm{zu}$ verhalten (Bundesministerium für Gesundheit 2008). Wenn eine der Bedingungen nicht erfüllt ist, beträgt die Zuzahlungsgrenze 2\%, und entspricht der Belastungsgrenze, die für alle anderen Versicherten gilt (Gemeinsamer Bundesausschuss 2007). Vor den Reformen von 2007 betrug die Grenze für alle Chroniker 1\%. Die 1\%-Schwelle ist weiterhin für Chroniker gegeben, die nicht an einer der drei genannten Krankheiten leiden. Um die Diskussion zu fokussieren, wird im Folgenden nur die Darmkrebs-Situation berücksichtigt, und die anderen beiden Krebsarten werden ausgeblendet. 


\section{Evidenz, Begründung und Durchführbarkeit}

In Deutschland ist Krebs ist die zweithäufigste Todesursache, und Darmkrebs stellt sowohl für Frauen wie für Männer den zweithäufigsten bösartigen Tumor dar. Die Inzidenz nimmt mit dem Alter zu, und für 2011 wurden 73.000 neue Fälle projiziert, darunter geschätzte 26.000 Todesfälle (Robert Koch-Institut 2010: 36). Die Mortalität schreitet relativ langsam voran: ein Jahr nach der Diagnose beträgt die Überlebensrate $83 \%$, nach fünf Jahren $65 \%$, nach 10 Jahren hat sie auf $58 \%$ abgenommen. Im Fall von frühzeitig lokalisiertem und behandeltem Darmkrebs kann sich die Überlebensrate auf $90 \%$ verbessern, allerdings werden aufgrund niedriger Screeningraten relative wenige Erkrankungen in diesem Stadium erkannt (American Cancer Society 2011: 7).

Dem Nationalen Krebsplan zufolge wurden präventive Koloskopien, die seit 2002 angeboten werden, von 17\% der Frauen und von 15\% der Männer genutzt. Um die Teilnahme-Raten zu erhöhen, betont der Nationale Krebsplan, dass konzertierte Anstrengungen aller Beteiligten erforderlich sind, damit Anspruchsberechtigte »eine informierte Entscheidung zur Teilnahme treffen ... können « (Bundesministerium für Gesundheit 2011: 8).

Nach neueren Evaluationen wird geschätzt, dass Koloskopien für den Achtjahreszeitraum 2002-2010 ca. 99.000 Darmkrebs Erkrankungen verhindert haben, und in ca. 47.000 Fällen zur Entdeckung von behandelbaren Erkrankungen im Frühstadium geführt haben (Brenner, Altenhofen, und Hoffmeister 2010: 756). Der Nationale Krebsplan zitiert Forschungsergebnisse, denen zufolge die Mehrheit der Darmkrebs-Todesfälle verhindert werden könnten, wenn alle Anspruchsberechtigten das Screening-Angebot nutzen würden (Winawer et al. 1993; Sieg und Brenner 2007). Skeptiker weisen jedoch darauf hin, dass es bisher keine Daten zum Nutzen von Koloskopien aus randomisierten kontrollierten Studien (RCTs) gibt, und dass selbst bei hohen Raten der Nutzung nur drei bis vier Todesfälle pro 1.000 gescreenten Patienten verhindert werden können (Mühlhauser 2007: 1806, vgl.; Mühlhauser 2011). Ein ähnliches Spektrum an Einschätzungen ergab sich aus den im Rahmen dieser Studie durchgeführten Interviews mit Ärzten. Mehrere Onkologen sprachen insbesondere das Fehlen von Evidenz aus RCTs an. Ich bin nicht qualifiziert, die Frage der tatsächlichen Evidenz zu bewerten, und fahre hier unter der Annahme fort, dass, selbst wenn derzeit kein Konsens zur Größe des Nutzens besteht, es plausibel ist anzunehmen, dass Koloskopien das Risiko an Darmkrebs zu sterben für einen nicht unbeträchtlichen Teil der Anspruchsberechtigten in relevanter Weise senken können.

Es ist ebenso unklar, wie die ökonomische Rechtfertigung ausfällt, die signifikant von der Klärung der Frage abhängt, wie viele Anspruchsberechtigte Koloskopien nutzen müssen, um eine bestimmte Zahl von Todesfällen zu verhindern, oder Leben um eine bestimmte Zeit zu verlängern. Bezüglich der Ausgabenseite betont der Nationale Krebsplan, dass Krebstherapien Ressourcenund kostenintensiv sind: Für eine sechsmonatige medikamentöse Standardtherapie bei Patienten in fortgeschrittenen Stadien (III und IV) betragen die Kosten ca. 40.000 €, und es wird angenommen, dass die Verfügbarkeit neuer Biologika $\mathrm{zu}$ einer weiteren Erhöhung auf bis zu $250.000 €$ pro Fall führen könnte (Bundesministerium für Gesundheit 2011: 1). In welchem Ausmaß diese Kosten durch Screening vermieden werden können, ist unmittelbar vom Verhältnis der medikamentösen Kosten zu den Kosten der Implementierung des Screenings abhängig. Es ist bemerkenswert, dass weder der Kommentar der Bundesregierung, der die Einführung der Reform 2007 begleitete (Bundesregierung 2006), noch der

\section{Die Wirtschaftlichkeit der Darmkrebsvorsorge wird schlicht als erwiesen angenommen.}

Nationale Krebsplan solche (und weitere relevante) Daten bereitstellen. Die Wirtschaftlichkeit wird schlicht als erwiesen angenommen.

Normativ kann der Umstand möglicher Einsparungen durchaus angeführt werden, um an die Eigenverantwortung der gesetzlich Versicherten zu appellieren, wie dies auch in $\mathbb{S} \mathbb{S} 1$ und 2 SGB V angelegt ist, und ebenso von der damaligen Gesundheitsministerin Ulla Schmidt bei der Einführung der Gesundheitsreform 2007 betont wurde: »Wenn Krankheiten frühzeitig erkannt werden, können sie besser behandelt und in vielen Fällen geheilt werden. Deshalb bietet die gesetzliche Krankenversicherung allen Versicherten eine Reihe von Vorsorgeund Früherkennungsuntersuchungen an. Ich wünsche mir, dass mehr Menschen dieses Angebot wahrnehmen. Mit der Gesundheitsreform haben wir dafür neue Anreize geschaffen. Mir ist wichtig, dass jeder und jede mehr Verantwortung übernimmt: für sich selbst, aber auch für die Versichertengemeinschaft, die die Kosten für die Behandlung tragen muss." (Bundesministerium für Gesundheit 2007)

Vorläufig kann festgehalten werden, dass ein entscheidendes Element der Rechtfertigung der Revidierung des $\$ 62$ SGB V bezüglich des Potenzials an Kosteneinsparungen nicht geliefert wurde, jedoch vernünftigerweise eingefordert werden könnte. Dies ist auch deswegen relevant, weil die Regelung einen gänzlich anderen Charakter annehmen würde, wenn das ökonomische Argument nicht haltbar wäre. Ein negatives Ergebnis hätte insbesondere Implikationen für die Frage, wie weit Maßnahmen gerechtfertigt sind, die über reines Anraten hinaus gehen, und damit ein stärkeres paternalistisches Element beinhalten. In der Abwesenheit von klarer Evidenz, dass die Teilnahme einer hinreichend großen Anzahl von Anspruchsberechtigten am Darmkrebscreening positive Auswirkungen auf die mit den Handlungsoptionen verbundenen Kosten hat, gehe ich im Folgenden hier dennoch davon aus, das sich eine günstige Prognose ergibt. Selbstverständlich würde sich in der Schlussbilanz ein anderes Bild ergeben, wenn dies nicht der Fall sein sollte.

Bezüglich der Begründung von der Seite der damaligen Bundesregierung

bei der Einführung des Gesetzes ist weiterhin bemerkenswert, das sich auch keine explizite Rechtfertigung der Anforderung des ursprünglich wesentlich weiter gehenden "therapiegerechten Verhaltens « fand. Nach einer hitzigen Schluss-Debatte im Bundestag am 2. Februar 2007 wurde der G-BA beauftragt, die Umsetzung zu konkretisieren, wobei diese Bestimmung in gewisser Weise ab- 
gemildert wurde, indem verlangt wurde, die Behandlung nicht abzulehnen (statt sich in jeder Hinsicht konform zu verhalten). Dies war angesichts der gesetzlich bereits verabschiedenden Rahmenbedingungen das äußerst Mögliche. Das übereilte Verfahren und der große Zeitdruck, wie auch die unzureichende Einbindung relevanter Interesseneigner unterminieren in signifikanter Weise die Legitimität der Regelung.

In praktischer Hinsicht ist die Implementierung der Beratungs-Regelung durchaus durchführbar, solange systematisch dokumentiert wird, wer wann an entsprechenden Gesprächen teilgenommen hat. Das zweite Element bezüglich der Erfordernis, medizinisch angeratene, bzw. zu einem bestimmten Zeitpunkt angenommene Behandlung nicht abzulehnen, kann hingegen auf beträchtliche Hindernisse stoßen, die weiter unten aufgenommen werden.

\section{Stärke des Eingriffs und Zwang}

In den hier durchgeführten Interviews und Umfragen wurde die Chronikerrichtline nur auf Darmkrebs bezogen, und mit der Aussage abgeschlossen: »Eine Krankenkasse sagt, dass die Regelung angemessen sei, da DarmkrebsPatienten, die die beiden Bedingungen erfüllen, helfen, Kosten zu kontrollieren." ̈̈rzte wurden in einer offenen Frage um ihre Einschätzung gebeten, und die Teilnehmer der Online-Umfrage bekamen eine fünfpolige Likert-Skala, um ihre Einschätzung abzugeben. Es ist bemerkenswert, dass nicht ein einziger der befragten Ärzte die Chronikerrichtlinie in der gegenwärtigen Form uneingeschränkt begrüßte, wohingegen 37\% der Teilnehmer der Umfrage »stimme $\mathrm{zu}$ « oder »stimme voll und ganz $\mathrm{zu}$ « wählten (in der Folge werden beide Optionen zusammenfassend unter "stimme zu « wiedergegeben). $28 \%$ wählten die neutrale Option (»stimme weder zu, noch lehne ich $a b \ll)$ und $36 \%$ wählten »stimme nicht zu« bzw. »stimme überhaupt nicht $\mathrm{zu}$ « ( $\mathrm{n}=983)$. Das heißt, dass $65 \%$ der Befragten die Regelung und die dargestellte Begründung entweder akzeptieren oder begrüßten (Vgl. Koch und Scheibler: 190).

In der Alterszielgruppe (50-65 Jahre, $\mathrm{n}=336$ ), ergab sich ein ähnliches Bild, mit etwas höherer Ablehnung: 37\% stimmten $\mathrm{zu}, 22 \%$ wählten die Mittelpositi- on, und $41 \%$ lehnten die so begründete Regelung ab. Das Geschlecht war nur in der Gesamtgruppe signifikant, wobei Frauen weniger zustimmten und höhere Ablehnung zeigten (Zustimmung: 32\% vs. $41 \%$, Ablehnung: $40 \%$ vs. $31 \%, c^{2}[4$, $\mathrm{n}=983]=13.90$, p.0076). In Bezug auf die Einkommen zeigte die mittlere der drei Gruppen $^{2}$ die höchste Ablehnung (von niedrig-hoch: $32 \%, 38 \%, 25 \%, c^{2}[8$, $\mathrm{n}=983]=15.54$, p.0495), mit einem parallelen, jedoch ausgeprägterem Muster in der Alters-Zielgruppe (von niedrig-hoch: 28,50 , und $27 \%, \mathrm{c}^{2}[8, \mathrm{n}=336]=21.28$, p.0064). Es ergaben sich weiterhin signifikante Unterschiede nach dem (selbst berichteten) Gesundheitsstatus: in der Gesamtpopulation der Umfrage verteilte sich die Ablehnung wie folgt: schlecht: $57 \%$, weniger gut: $43 \%$, gut: $35 \%$, sehr gut: $29 \%$, ausgezeichnet: $42 \%$; $\left(c^{2}[16\right.$, $\mathrm{n}=961]=53.33$, p.<.0001, in der Zielgruppe waren $\mathrm{c}^{2}$ suspekt aufgrund der kleineren $\mathrm{Zahl}$ ).

In Bezug auf die Nutzung von Darmkrebsvorsorge zeigte sich, dass 35\% derjenigen, die bereits eine Koloskopie hatten die so begründete Regelung ablehnten, $27 \%$ derjenigen, die andere Screeningformen genutzt hatten, und $37 \%$ derjenigen, die keinerlei Screening hatten. In der Zielgruppe war dieses Muster ausgeprägter $(40 \%$ vs. $27 \%$ und $48 \% ; c^{2}[8, n=967]=17.98$, p.0214; Zielgruppe [5065]: $c^{2}[8, n=330]=16.45$, p.0364).

In den Interviews sagte der einzige Arzt, der die Regelung mit Einschränkungen akzeptierte: "Naja, des Menschen Wille ist sein Himmelreich ... jeder sollte snein sagen können, wenn er will. Auf der anderen Seite basieren die Kassen auf dem Solidarprinzip, und die, die sich nicht solidarisch verhalten, müssen mehr zahlen ... ökonomisch macht die Regelung Sinn. Aber vom Psychologischen her finde ich Bonus besser als Malus." Die Opposition zur Regelung war insbesondere im Compliance-Erfordernis begründet. Auf unterschiedliche Weise artikulierten viele Ärzte die Meinung, dass diese Patienten doppelt bestrafte, da sie zu der Krankheit und der nachvollziehbar oft schwierigen Entscheidung für oder gegen aggressive Therapien zusätzlich die Belastung hätten, die Folgen höherer Kosten zu erwägen. Eine Ärztin fand es daher "wirklich extrem " die Beratungs- und Compliance-Regelung auf diese Weise zu verbinden. Ebenso wurde die Effektivität in Bezug auf typisches menschliches Verhalten in Frage gestellt: »Ich glaube einfach nicht, dass Patienten soweit voraus denken... Leute wollen sich einfach nicht mit der Möglichkeit [dass sie Darmkrebs bekommen] beschäftigen, und gehen deswegen nicht zum Screening. Deshalb glaube ich, dass die Bestrafung hier wirklich nicht sinnvoll ist."

Diese Ansichten haben beträchtliche Plausibilität. Die Entscheidungsfindung in derartigen Situationen ist schwierig genug. Zu verlangen, dass Patienten zusätzlich die finanziellen Auswirkungen berücksichtigen, kann zu unangemessener Intrusion und Belastung führen. Weiterhin ist unter rein ökonomischen Gesichtspunkten auch nicht ohne weiteres ersichtlich, warum Patienten dazu bewegt werden sollten, sich Kosten verursachenden Behandlungen zu unterzie-
Aufgrund ethischer und ökonomischer Erwägungen spricht wenig dafür, die niedrigere Zuzahlungsgrenze an das Annehmen medizinisch angeratener Behandlung zu knüpfen.

hen, wenn sie andernfalls (sehr wahrscheinlich) früher sterben würden, und damit weniger Kosten verursachen. Sowohl aufgrund ethischer wie auch ökonomischer Erwägungen spricht daher wenig dafür, die niedrigere Zuzahlungsgrenze an das Annehmen medizinisch angeratener Behandlung zu knüpfen.

\footnotetext{
2 Es wurden drei in der Armutsforschung übliche Gruppen gebildet: bis zu $60 \%$ des medianen Brutto-Haushalteinkommens (niedrig), 60-200\% (mittel), und über 200\% (hoch). 2006 medianes Brutto-Haushalteinkommen (HHE) war $32.048 €$. Die niedrige Einkommensgruppe mit $<60 \%$ HHE umfasste bis zu $19.228 €$ (gerundet auf $<20,000 €$ ); die mittlere Gruppe $19.229 €-64.096 €$ (gerundet auf $20.000 €-64.999 €$ ), und die Gruppe mit hohem Einkommen bezog sich auf alle über $65.000 €$.
} 
Was die Intrusion des BeratungsErfordernis angeht, sind zwei Aspekte hervorzuheben, die mit der Weise zu tun haben, wie der Anreiz geschaffen wurde (Verdopplung der Zuzahlungsgrenze), und der Aktivität, für die Anreize geschaffen wurden (über Darmkrebs nachdenken). Prinzipiell hätte ein Anreiz auch dadurch geschaffen werden können, dass die Zuzahlungsgrenze vermindert worden wäre. Auf diese Weise hätten diejenigen, die die Regel befolgen, eine Grenze von 0,5\% statt $1 \%$, was zu einer anderen Wahrnehmung, und auch einer anderen finanziellen Belastung geführt hätte. Die Forschung der Verhaltensökonomik legt zwar nahe, dass das Vermeiden von Verlusten ein stärkerer Motivator sein kann als die Möglichkeit, Gewinne zu machen (Kahneman und Tversky 1979), aber es ist bemerkenswert, dass eine derartige Rechtfertigung (oder etwa positive Ergebnisse von Pilot-Studien) weder in der Begründung des Gesetzes (Bundesregierung 2006), noch in den folgenden Debatten oder Dokumenten angeführt wurden. ${ }^{3}$ Obwohl die Mechanik der Regelung daher in einer Verdopplung des Grundbetrags bestand, verneinten die Proponenten vehement, dass dieses Element einen Nachteil darstellte, wie beispielsweise aus der Behauptung Jens Spahns MdB, CDU, ersichtlich wird, der in der Bundestagsdebatte gegenüber der damaligen Opposition argumentierte: »Es handelt sich also nicht, wie Sie es fälschlicherweise seit Wochen und Monaten nennen, um eine 'Strafzahlung. Es ist vielmehr so, dass man die entsprechende Vergünstigung nicht bekommt." (Deutscher Bundestag 2007: 8036)

Während drei der befragten Ärzte dennoch mit dieser Sichtweise (und dem Tenor der oben zitierten Ulla Schmidt) übereinstimmten, und die Regelung als positiven Anreiz auffassten, sich gesundheitsförderlich zu verhalten, empfanden vier sie nur als negative Bestrafung. Zwei fanden, dass die Regelung beides zugleich darstellte, und ein Arzt fand, dass keine dieser Sichtweise zutreffend sei. In der Umfrage ergab sich, dass $32 \%$ die Regelung eher als Belohnungsprogramm empfanden, $33 \%$ als Bestrafungsprogramm, und $35 \%$ die sowohl-als-auch Option wählten: etwa zwei Drittel nehmen also ein Bestrafungselement wahr. Altersunterschiede, Einkommen und Nutzung der Darmkrebsvorsorge wa-

Abb. 2: Umfrageergebnisse - Akzeptanz dreier Anreiz-Szenarien zur Förderung der Darmkrebsvorsorge (in \%)

\begin{tabular}{|l|c|c|c|} 
& $\begin{array}{c}\text { Beratung und } \\
\text { Compliance } \\
(n=983[336])\end{array}$ & $\begin{array}{c}\text { Beratung } \\
(n=976,[333])\end{array}$ & $\begin{array}{c}\text { Screening } \\
(n=974,[333]\end{array}$ \\
\hline Stimme überhaupt nicht zu & $17[20]$ & $16[22]$ & $15[21]$ \\
\hline Stimme nicht zu & $19[21]$ & $15[14]$ & $21[22]$ \\
\hline $\begin{array}{l}\text { Stimme weder zu, noch leh- } \\
\text { ne ich ab }\end{array}$ & $28[22]$ & $29[22]$ & $29[21]$ \\
\hline Stimme zu & $27[24]$ & $29[31]$ & $27[29]$ \\
\hline Stimme voll und ganz zu & $10[13]$ & $10[11]$ & $8[7]$ \\
\hline Zahlen in Klammern stellen Werte für die Zielgruppe [50-65 Jahre] dar. \\
\hline
\end{tabular}

ren nicht signifikant, allerdings sahen etwa 10\% mehr Männer als Frauen die Regelung als Belohnungsprogramm an (37\% vs. $26 \%, c^{2}[2, n=975]=12.23$, p.0022). Bezüglich des Gesundheitszustands waren Gesündere eher geneigt, dem Belohnungsmodell zuzustimmen, jedoch waren die Unterschiede statistisch nicht signifikant.

In zwei weiteren Szenarien wurden Einstellungen zu abgewandelten Regelungen der Chronikerrichtline untersucht. ${ }^{4}$ Im ersten Fall ging es lediglich um das Informationselement: Versicherte erhalten einen einmaligen Prämienaufschlag von $200 €$, wenn sie nicht innerhalb von drei Jahren nach Erhalt eines Einladungsschreibens zu einem Beratungsgespräch über die Vor- und Nachteile der Darmkrebsvorsorge kommen (im Folgenden wird dieses Szenario `Beratungs-Regelung (genannt). Im zweiten Fall ging es um das im GKVWSG ursprünglich geplante Vorhaben, den Anreiz direkt an die Durchführung eines Screenings zu binden: Versicherte erhalten einen einmaligen Prämienaufschlag von $200 €$, wenn sie nicht innerhalb von drei Jahren nach Erhalt eines Einladungsschreibens die Untersuchung durchführen lassen (im Folgenden wird dieses Szenario 'Screening-Regelung genannt). Bemerkenswerterweise zeigen sich nur sehr geringe Unterschiede in der Bewertung der drei sehr unterschiedlich gelagerten Szenarien, sowohl in der Umfragepopulation insgesamt, als auch in der Zielgruppe der 50-65-Jährigen, wie aus Abbildung 2 ersichtlich.

In den Interviews mit den Ärzten ergab sich jedoch an anderes Bild. ${ }^{5}$ Während nur ein Arzt die Chronikerrichtline (mit Einschränkungen) akzeptabel fand, fanden drei Ärzte die Screening-Regelung annehmbar (wenngleich zwei mit Einschränkungen), und fünf konnten der Beratungs-Regelung zustimmen (zwei mit Einschränkungen). Die Gegner der Screening-Regelung betonten zentral das Recht auf Nichtwissen (das auch im Beschluss des G-BA hervor gehoben wurde). Die Befürworter stellten hingegen auf Solidarität und gesundheitliche Nutzen ab: »Der Ansatz hier ist eben, Kooperation zu bewirken... das ist ja auch in der Präambel des Sozialgesetzbuchs, dass Versicherte dazu beitragen sollen, Krankheit zu verhindern ", »Wir reden hier über Darmkrebs. Da ist es schon in Ordnung, Leute gewissermaßen zu ihrem Glück zu zwingen «, »Ich glaube, das können wir den Versicher-

\footnotetext{
3 Es wurde auch nicht gezeigt, dass eine Absenkung der Zuzahlungsgrenze über ein Modell der Gewinnbeteiligung nicht möglich wäre, weil die damit verbundenen geringeren Beiträge sich negativ zu den Einsparungen verhalten, die aus der Screening Teilnahme ergeben - obwohl, wie oben ausgeführt, von beträchtlichen Effizienzen ausgegangen wird. Zum Teil kann die Abneigung das Absenkungsszenario zu erwägen, auch vor dem Hintergrund verstanden werden, dass zur Zeit etwa 40\% der Versicherten die Zuzahlungsgrenze erreichen, und einige Kommentatoren argumentieren, dass diese Situation es unabhängig von Anreizprogrammen erfordert, die Schwelle anzuheben (Ich danke Prof. Jürgen Wasem für die Klärung dieses Punktes, pers. Korrespondenz, 22. Juli 2011)

4 Die Fragen folgten direkt auf die beiden vorgenannten Fragen, wurden in der Abfolge jedoch randomisiert.

5 Im Gegensatz zu den Umfrage-Szenarien, bei denen der Anreiz eine Aufzahlung von $200 €$ drei Jahre nach Anspruchsberechtigung war, wurde im Fall der Interviews (die der Umfrage zeitlich vorausgingen) der Anreiz der Chronikerrichtlinie verwendet, d. h. $1 \%$ statt $2 \%$ Zuzahlung. Die Funde können daher nicht direkt verglichen werden, sind aber dennoch ähnlich.
} 
ten zumuten. Die GKV funktioniert nun mal auf dem Solidarprinzip, wir zahlen alle ein, und sollten den Schwachen und Armen helfen, aber es kann nicht sein, dass einige nur Trittbrett fahren. Und solche Regelungen können das verhindern.«

Die Befürworter der ScreeningRegelung stimmten durchweg der Beratungs-Regelung zu, allerdings waren zwei Gegner der ScreeningRegelung auch bereit, die Beratungs-Regelung $\mathrm{zu}$ akzeptieren: »Also hier stimme ich zu ... wenn es wirklich nur um Beratung geht. Wir sind eben in einem Solidarsystem... und das ist auf so einem niedrigen Level ... da erwartet man wirklich nicht zu viel. Man übertreibt es mit der Freiheit, wenn man sagt, das geht nicht. "Für die Gegner standen normative und praktische Gründe im Vordergrund: "Selbst wenn es nur ums Nachdenken geht... ich finde, dass kann man nicht zur Pflicht machen. Das geht einfach gegen meine grundlegenden ethischen Instinkte." »Wie will man denn Beweisen, dass die Leute auch wirklich nachgedacht haben? [...] Und andersrum, bloß weil jemand nicht zur Beratung gekommen ist, heißt das doch nicht, dass die nicht nachgedacht haben - die können ja auch nen Fall in der Familie haben, oder sich sonst wo informieren, und wissen sehr wohl worum es geht ... . Man muss auch mal sehen, was hinter diesen Regelungen steht: da geht es um Strukturen, und darum Geldflüsse zu regeln, nicht nur darum, Krankheiten anders oder früher zu behandeln." »Ich würde das als Empfehlung machen: sollen: ja, aber müssen: nein ... irgendwo muss auch Schluss sein."

Es ist richtig, dass die BeratungsRegelung in relevanter Weise neu und einzigartig ist. Es gibt derzeit keinen anderen Bereich, in dem Versicherte nachweisen müssen, über die Vor- und Nachteil bestimmter Optionen nachgedacht haben, mit der Ausnahme der Abtreibungsregelung (wobei es hier der Rechtfertigung nach um die Abwägung der Interessen zweier Träger geht). In der Darmkrebs-Beratungs-Regelung kommen die Interessen anderer nur indirekt ins Spiel, über die Implikationen, die die individuelle Entscheidung für den Ressourcen-Pool der solidarischen Versicherungsgemeinschaft hat.

Natürlich muss an dieser Stelle erin- nert werden, dass die >Pflicht zum Denken nicht ursprünglich im GKV-WSG angelegt war, sondern sich lediglich aus der Umsetzung des G-BA ergab, der die kontroverse Bestimmung im Rahmen der gesetzlichen Vorgaben nicht weiter dass sie Wahlmöglichkeit implizieren, d. h. die Regel gilt nur für Versicherte, die das Alter der Anspruchsberechtigung erst noch erreichen. So offensichtlich dieser Ansatz auch erscheinen mag, kann er doch nicht vorausgesetzt werden, da z. B. \$ 52 SGB V, der ebenso unter dem GKV-WSG reformiert wurde, es GKVen ermöglicht, im Fall von Krankheiten die sich aus einer »medizinisch nicht indizierte[n] Maßnahme, wie zum Beispiel eine[r] ästhetische[n] Operation, eine[r] Tätowierung oder ein[es] Piercing[s] « ergeben, die

abmildern konnte. Nichtsdestotrotz kann argumentiert werden, dass, insofern die medizinische und ökonomische Evidenz robust ist, und das Potenzial anderer Ansätze, wie etwa aktiver Einladungsverfahren, erschöpft ist, ${ }^{6}$ die Regelung nicht lediglich einen tolerablen politischen Kompromiss darstellt, sondern, prinzipiell, eine akzeptable, wenngleich auch offenkundig paternalistische Regelung sein kann, Gesundheitsmündigkeit (Wuppermann, Wuppermann, und Riemann 2009), Eigenverantwortung und Gesundheit auf der Einzel- und Populationsebene zu befördern.

Bezüglich Intrusion und Zwang kann im Zwischenfazit festgestellt werden, dass die Konzepte auf die Chronikerrichtlinie zutreffen, wie auch auf die beiden hier explorierten Variationen. Die Regelungen beziehen sich auf Erwägungen, die plausibler Weise in den privaten Bereich fallen, und zudem wurde der Anreiz durch die Vermeidung einer Erhöhung umgesetzt, und nicht durch das Anbieten einer effektiv geringeren Belastung.

\section{Zuschreibbarkeit/ Handlungsmöglichkeit}

Nach dem Diktum »Sollen impliziert können « ist es wenig hilfreich, wenn nicht zynisch, Menschen Verpflichtungen zuzuschreiben, wenn sie diese nicht erfüllen können. Für die hier untersuchte Regelung ist das in verschiedener Hinsicht relevant. Erstens wäre es kafkaesk, die Chronikerrichtlinie retrospektiv für eine beispielsweise 70 -jährige Patientin umzusetzen, die dringend eine Darmkrebsoperation benötigt, jedoch vor 20 Jahren keine Beratung zum Thema hatte. Aus diesem Grund sind, wie oben erwähnt, die Alters-Kriterien so gewählt,
Versicherten »in angemessener Höhe« an den Kosten zu beteiligen, und Krankengeld ganz oder teilweise zurückzufordern - hier schien es dem Gesetzgeber gleichgültig zu sein, dass die Versicherten zum Zeitpunkt, da sie die anstößigen Handlungen vollzogen, nicht wissen konnten, dass diese in einem Nachteil resultieren könnten.

Die temporale Dimension ist gleichwohl im Darmkrebsfall in anderer Hinsicht relevant. Stellen wir uns einen 55-jährigen Mann vor, der gesund ist, von der Regelung weiß, aber nicht geneigt ist, am Beratungsgespräch teilzunehmen, vielleicht weil er insgesamt wenig Gesundheitsprobleme und auch keine Vorgeschichte von Darmkrebs in der Familie hat, und nicht glaubt, dass ihm die $2 \%$ statt der $1 \%$ Zuzahlungsgrenze finanzielle Schwierigkeiten bereiten würde. Zwanzig Jahre später ist er wesentlich gesundheitsbewusster

\footnotetext{
6 Wiederum ist bemerkenswert, dass die Bundesregierung in der Einführung nicht argumentierte, dass alle plausiblen Alternativen erschöpft worden (oder zumindest erwägt worden) wären. Beispielsweise zitiert der Nationale Krebsplan Ergebnisse von Pilotprojekten im Saarland, denen zufolge ein Einladungsschreiben mit einer Liste qualifizierter Ärzte zu einer Verdoppelung der durchgeführten Koloskopien geführt hat, und ein ähnliches Verfahren der AOK in Hamburg und Rheinland-Pfalz führte zu einer Zunahme gegenüber dem Vorjahr um 75\% (in beiden Fällen wurden keine Angaben zu den absoluten Basis- oder Endwerten gemacht (Bundesministerium für Gesundheit 2011: 10). Ebenso ist bemerkenswert, dass die Raten in anderen Ländern wesentlich höher sind, in den USA um etwa $30-40 \%$, da $48 \%$ der anspruchsberechtigten Frauen und 52\% der Männer innerhalb der letzten 10 Jahre eine Koloskopie oder innerhalb der letzten 5 Jahre eine Sigmoidoscopie in Anspruch nahmen (American Cancer Society 2011: 14). Es wäre sicherlich sinnvoll zu verstehen, was die Determinanten solcher beträchtlichen Variation sind.
} 
geworden, seine finanzielle Situation hat sich drastisch verschlechtert, und er benötigt eine Darmkrebsoperation. Darauf zu bestehen, dass er die höhere Zuzahlungsgrenze zu tragen hat, liegt offensichtlich im Rahmen des Gesetzes. Dennoch kann hier - auch angesichts der von den Ärzten im Interview angemahnten >doppelten Bestrafung', und des Umstandes, dass etwa 40\% der Umfrageteilnehmer die Regelung ablehnen - fraglich sein, ob die Bestimmungen ihr Ziel erfüllen, und ob es nicht bessere Wege gibt, es zu erreichen.

Wie bereits erwähnt, ist die öffentliche Rechtfertigung der Regelung dürftig. In einer wohlwollenden Auslegung könnte man rekonstruieren, dass sie auf Gerechtigkeitserwägungen beruht. Denn die Strafe betrifft nur diejenigen, die auch erkrankten, und daher (im besten Fall) den Schaden hätten abwenden können, was gegebenfalls ein »Modell kostenüberwälzender (retrospektiver) Rechenschaftsverantwortung « rechtfertigen kann (Höfling 2009: 291). Aber es wäre ebenso möglich, weniger auf die tatsächlichen Konsequenzen des Handelns zu fokussieren, und mehr auf die Risiken, die mit bestimmten Handlungen assoziiert sind, etwa, nicht an einem Beratungsgespräch teilzunehmen (Cappelen und Norheim 2005: 478; Le Grand 1991: 121).

Weiterhin spricht auch die Forschung der Verhaltensökonomik und Prospekttheorie dafür, den Zeitpunkt des Anreizes (ob dieser in der Vermeidung einer Bestrafung oder dem Erhalt einer Belohnung besteht) vorzuverlegen, da weiter in der Zukunft stattfindende Ereignisse generell als weniger real und handlungsleitend empfunden werden (Loewenstein, Brennan, und Volpp 2007; Frederick 2002). Für den hier vorliegenden Zusammenhang ist es plausibel anzunehmen, dass Versicherte einen Nachteil, der sich relativ unmittelbar einstellt, als Anreiz ernster nehmen, als einen, der nur dann eintritt, wenn sie eine Erkrankung erleiden (die generell verdrängt wird), und die sich noch dazu erst Jahrzehnte nach der ursprünglichen Anspruchsberechtigung manifestieren kann. Obwohl es daher eine gewisse Plausibilität hat, den Anreiz direkt damit zu verbinden, ob jemand tatsächlich an einer verhinderbaren Krankheit leidet, steht die Mechanik der Chronikerrichtlinie doch in großer Spannung zum
Stand der Forschung in der Verhaltensökonomik, und weist zudem das Risiko auf, Menschen in einer verletzlichen Situation zusätzlich zu belasten.

Ein weiterer und damit verwandter Punkt hat mit der Annahme zu tun, dass alle Versicherten von der Regelung wissen. Wenngleich die Bestimmungen in SGB V und die folgende Konkretisierung durch den G-BA deutlich sind, sind einzelne GKVen derzeit aufgrund von Datenschutzbestimmungen generell nicht berechtigt, Versicherte gezielt anzuschreiben, einzuladen, und auf die Konsequenzen hinzuweisen, wenn sie sich nicht entsprechend verhalten. Insbesondere können sie nicht Versicherte kontaktieren, die beispielweise in den letzten beiden Jahren keine präventiven Leistungen in Anspruch genommen haben. ${ }^{7}$ Sie können vielmehr nur indirekt und in allgemeiner Form informieren, etwa über die Mitgliedszeitschriften oder elektronische Newsletter. Dieser Umstand ist wenig hilfreich, und sollte in jedem Fall revidiert werden, um sicher zu stellen, dass alle Versicherten in Kenntnis der (singulären) Regelung sind. Weiterhin ist ebenso erforderlich, dass die Beratung in nicht-direktiver Weise durchgeführt wird (Koch und Scheibler, 2007: 190-194), und ohne dass es zu Interessenkonflikten für etwa beteiligte Ärzte kommen kann.

\section{Gerechtigkeit}

Inzidenz und Prävalenz des Darmkrebs, wie auch die Nutzung der Darmkrebsvorsorge kann über verschiedene Regionen, Alters-, Einkommens-, oder Bildungsgruppen hinweg variieren. In einer Umfrage unter Deutschen, die älter als 50 Jahre waren (Wahrscheinlichkeitsstichprobe, $n=1,921)$ untersuchten Olaf von dem Knesebeck und Andreas Mielck Unterschiede in der Nutzung präventiver Leistungen über je drei Gruppen in den Kategorien Bildung, Äquivalenzeinkommen und Geldvermögen. Bezüglich der Inanspruchnahme von Koloskopien zeigte sich, dass Menschen mit einem vergleichsweise hohen sozialen Status eine höhere Wahrscheinlichkeit aufwiesen, Koloskopien zu nutzen. (Odd Ratios und 95\% Konfidenzintervalle in aufsteigender Reihenfolge bezüglich Sta- tus waren, für Bildung: 1,0, 1,20 (0,891,62), 1,48 (1,06-2,07), für Äquivalenzeinkommen: 1,0, 1,02 (0,80-1,30), $0,94(0,74-1,20)$, für Geldvermögen: 1,0, 1,32 (1,04-1,69), 1,36 (1,07-1,74) von dem Knesebeck und Mielck 2009: 45-48).

\section{Es ist illusorisch zu erwarten, strikte Gleichheit in Nutzung von Vorsorge- .}

Es ist generell illusorisch zu erwarten, dass strikte Gleichheit in der Nutzung von Vorsorgeuntersuchungen möglich ist. Dennoch ist es wichtig, Unterschiede in der Nutzung zu ermitteln, denn wo sie nicht darauf beruhen, dass Personen sich freiwillig für oder gegen verfügbare $\mathrm{Op}$ tionen entschieden haben, kann es sein, dass strukturelle Merkmale angepasst werden müssen, um Ungerechtigkeit zu vermeiden. Mit Blick auf die Daten von Knesbeck und Mielck kann man vermuten, dass die Chronikerrichtlinie für die sozial Bessergestellten keinen besonderen Anreiz bietet, da sie Vorsorgeuntersuchungen sowieso häufiger nutzen. Für die schlechtergestellten Gruppen zeigt sich wiederum, dass das Erfordernis der direkten Information an Wichtigkeit zunimmt, da diese Gruppen weniger gesundheitsmündig sind. Dieses Kriterium ist auch deswegen von Relevanz, da $\mathbb{} 20$ SGB V die GKVen explizit auffordert, zur Verminderung sozial bedingter Ungleichheit beizutragen. Es wäre sicherlich nicht erstrebenswert, wenn das Programm soziale Ungleichheiten der gesundheitlichen und wirtschaftlichen Nutzung vergrößert, statt verkleinert. Dies ist insbesondere auch in der Evaluation zu berücksichtigen.

\footnotetext{
7 Gemäß § 284 Abs. 3 SGB V dürfen die bei der Krankenkasse gespeicherten versichertenbezogenen Daten nur für Zwecke der Aufgaben nach § 284 Abs. 1 (Rechnungsprüfung) in dem jeweils erforderlichen Umfang verarbeitet und genutzt werden, für andere Zwecke, soweit dies durch Rechtsvorschrift des SGB angeordnet oder erlaubt ist. Ich danke Dirk Horenkamp-Sonntag für die Hilfe bei der Klärung dieser Frage.
} 


\section{Solidarität}

Wie bereits mehrfach in den Antworten der interviewten Ärzte aufschien, sind die Konzepte der Solidarität und Eigenverantwortung sowohl im übergreifenden $\mathbb{1} 1$ SGB V als auch konzeptuell eng verbunden. Diese Verschränkung allein ist jedoch keineswegs entscheidend für die Frage, ob die Chronikerrichtlinie gerechtfertigt ist. Diejenigen, die nicht mit der Regelung übereinstimmten, wiesen darauf hin, dass die Grenze der Belastung gerade von einer Interpretation des Solidaritätsgedanken gestützt werde, derzufolge man Hilfe leistet, weil andere sich in einer Situation von beträchtlichem Nachteil befinden. Entsprechend wurde argumentiert, dass es unfair sei, einigen chronisch Kranken Sonder-Bedingungen zu stellen: »Ich finde, es sollten alle [Chroniker] die gleiche Zuzahlungsgrenze haben. Vielleicht haben wir Gesunden dann etwas höhere Beiträge.... das ist das Solidaritätsprinzip - aber nicht, [einige] chronisch Kranke zu bestrafen!« Diejenigen, die die Chronikerrichtlinie, oder eine der abgewandelten Szenarien unterstützten, beriefen sich dagegen eher auf ein Konzept der Solidarität als der Reziprozität. Die Umfrage enthielt zu diesem Thema keine Frage, allerdings kann der Umstand, dass ca. $40 \%$ die Chronikerrichtline (die im Szenario explizit mit dem Kostenrational gerechtfertigt wurde) entweder befürworteten oder ablehnten, nahelegen, dass ähnliche Erwägungen eine Rolle gespielt haben.

Insgesamt lässt sich feststellen, dass die Regelung sowohl das Potenzial hat, Solidarität zu stärken, als auch zu unterminieren. Für das Argument der Stärkung der Solidarität ist allerdings ein stärkerer Nachweis als derzeit gegeben erforderlich, dass die Regelung tatsächlich zu Einsparungen führt, und die Ziele nicht auf andere Weise erreicht werden können. Dass das Netz der gegenseitigen Stützung für die Zielgruppen der Regelung im Gegensatz zu anderen Chronikern schwächer geworden ist, liegt hingegen auf der Hand.

\section{Wirkung auf Dritte}

Die Umfragen enthielten zwei Szenarien, in denen Teilnehmer gefragt wurden, ob sie ihren Arzt bitten würden, Mitteilungen bezüglich der Anreizprogramme an die GKV kontrafaktisch zu ihren Gunsten zu machen. Im ersten Fall ging es um ein Bonusprogramm, bei dem eine Person $150 €$ bekommen sollte, wenn sie bei zwei jährlichen Messungen einen normalen Body Mass Index (BMI) hatte: Im Szenario hatte eine Person bis zur ersten Messung $5 \mathrm{~kg}$ abgenommen, und den Zielwert erreicht, bei der zweiten lag sie jedoch $1 \mathrm{~kg}$ darüber, und bat den Arzt, das Erreichen des normalen BMI zu notieren. Im zweiten Fall ging es um die Chronikerregelung. Eine Patientin, die eine Darmkrebsoperation hatte, bat ihre Ärztin, keine adjuvante Chemo-Therapie durchzuführen, weil sie von einer Bekannten gehört hatte, dass diese sehr belastend sei, und nur wenig geholfen habe. Damit sie dennoch die niedrigere Zuzahlungsgrenze erhalten konnte, bat sie die Ärztin, der Krankenkasse zu melden, dass sie sich therapiegerecht verhielt.

Die Teilnehmer wurden gefragt zu schätzen, wie viele Patienten in solchen Situationen Ärzte bitten würden, unwahre Vermerke zu machen. Im BMISzenario wurde geschätzt, dass sieben von zehn dies täten (Mdn: 7, IQR=5-9, M: 6.52, SD=2.92, n=960). Die Werte für das Darmkrebsszenario lagen etwas darunter, bei fünf von zehn (sowohl in der Befragungspopulation insgesamt: Mdn: 5, IQR=3-8, M: 5, SD=2.99, $\mathrm{n}=941$, als auch in der Alters-Zielgruppe: $\mathrm{Mdn}: 5, \mathrm{IQR}=3-8, \mathrm{M}: 5, \mathrm{SD}=5.19$, $\mathrm{n}=114$ ).

Weiterhin wurde gefragt, wie die Handlung eines Arztes bewertet wird, der der Bitte eines Patienten nachgab (fünfpolige Likert-Skala von »hat sich völlig falsch verhalten « bis »hat sich völlig richtig verhalten«). In der Gesamtumfragepopulation fanden bezüglich des Darmkrebs-Szenario 14\%, dass sich der Arzt richtig verhalten hatte, 30\% wählten die neutrale Mittelposition, und $56 \%$ fanden das Verhalten falsch $(\mathrm{n}=941)$, mit stärkerer Ablehnung in der Alterszielgruppe (11, 22 und 67\%, $\mathrm{n}=114$ ).

In den Interviews mit den Ärzten ergab sich jedoch in relevanter Hinsicht ein anderes Bild. Bezüglich der quantitativen Frage wurden Ärzte gebeten zu schätzen, wie viele ihrer Kollegen wohl der Bitte eines Patienten in den jeweiligen Situationen nachkommen würden. Im BMI-Szenario schätzten neun der zehn Befragten, dass mehr als die Hälfte dies tun würden, was sich $>$ perfekt $<$ mit den Umfragewerten decken würde. Im Darmkrebsszenario hingegen meinten sieben, dass nicht ein einziger der Bitte nachgeben würde, zwei, dass einer, und einer, dass drei dies tun würden, ${ }^{8}$ womit eine weitaus größere Zahl Patienten das Nachsehen hätte, und ebenso eine größere Zahl Ärzte sich in einer unangenehmen Situation finden würde, einem Patienten in einer verletzlichen Lage die Bitte abschlagen zu müssen.

In den Interviews betonten mehrere Ärzte, dass das Darmkrebsszenario nicht realistisch sei, weil GKVen oft schon über Abrechnungsdaten informiert seien, und es weiterhin aus damit zusammenhängenden technischen und administrativen Gründen schwierig sei, dem Patientenwunsch zu entsprechen, von drastischen rechtlichen Konsequenzen einmal abgesehen. Dies ist selbstverständlich richtig, aber nicht notwendigerweise den Patienten klar, wie vielleicht auch aus den relativ hohen Umfragewerten geschlossen werden kann, denen zufolge jeder zweite Patient in einer solchen Situation den Arzt um Nachsicht bitten würde. Außerdem ist bemerkenswert, dass trotz dieser Einwände nicht alle Ärzte meinten, dass keiner ihrer Kollegen in einem solchen Fall der Bitte eines Patienten entsprechen würde, und es also für möglich halten, dass professionelle (und rechtliche) Standards zumindest gefährdet sein können. Zum Teil wurde dies mit der Nachvollziehbarkeit des Patientenwunsches begründet: »Ich hätte auch keine Lust auf Chemo - und wenn wir ehrlich sind, ist es ja schon so, dass nicht alle aus therapeutischen Gründen gemacht werden, sondern viele palliativ sind." Vor allem aber bekundeten Ärzte großes Unbehagen darüber, dass die Regelung, die ihnen rechtlich wenig Spielraum lässt, sie in die Situation bringt, eine Lüge erwägen zu müssen, und letztlich gegen die Interessen des Patienten zu handeln: »Hier geht es um Gesundheit, aber es geht auch um Profit für die Kassen. Und deswegen dann hier meine Schweigepflicht aufzugeben - da dreht sich mir echt der Magen um!«

\footnotetext{
8 Zur Vereinfachung der Darstellung wurde hier in Fällen, wo Zwischenwerte (z.B. $» 1-2 «)$ angegeben wurden, nach unten gerundet, da die Skepsis generell sehr hoch war.
} 
Absichten könne sich bekanntermaßen von tatsächlichem Verhalten unterscheiden. Zudem sind, wie oben angemerkt, die hier verwendeten $\mathrm{Me}$ thoden bezüglich ihrer Generalisierbarkeit begrenzt. Gleichwohl können diese Ergebnisse nahelegen, dass es durchaus plausibel ist, dass Patienten ihre Ärzte um unrichtige Mitteilungen an die Kassen bitten, und dass dies eine negative Auswirkung auf das Arzt-PatientenVerhältnis hat. Zusammen mit den sich oben ergebenden Bedenken spricht dies weiterhin dafür, das Compliance-Erfordernis in der gegenwärtigen Fassung der Chronikerrichtlinie abzuschaffen.

\section{Kohärenz}

Kumulativ kann daher nur ein Teil der Regelung bestehen. Gleichwohl stellt sich auch bei dem verbleibenden Element der Beratungs-Regelung die Frage, ob dieses in kohärenter Weise im weiteren Regelungszusammenhang einzupassen ist. Wie erwähnt, scheint es keine direkte Parallele zu geben, in der es eine Strafe für das Unterlassen des Nachdenkens über Vorsorgeuntersuchungen gibt. In dieser Hinsicht betritt die Regelung Neuland. Nichtsdestotrotz kann man sie prinzipiell durchaus von $\int 1$ SGB V gestützt sehen, oder implizit durch das Prinzip der RisikoVerteilung, das der Finanzierung der GKV unterliegt.

\section{Schluss}

Mit Bezug auf Evidenz, Begründung und Durchführbarkeit besteht kein Zweifel, dass Koloskopien vorzeitige Sterblichkeit verhindern können, wenngleich es sich nicht um eine Intervention handelt, der sich jeder gerne unterzieht. In Verbindung mit der Annahme, dass sich durch vermehrte Nutzung Kosten einsparen lassen, kann sich sicherlich eine prima facie-Verpflichtung für Versicherte ergeben, zu bedenken, ob sie die Untersuchung nutzen sollten. Aufgrund des Solidarprinzips kann diese Verpflichtung an Stärke gewinnen, je klarer die Evidenz zum Gesundheitsverbesserungs- und Kosteneinsparungspotenzial ist. Ob solche Verpflichtungen in der gegenwärtigen Form der Chronikerrichtlinie angemessen umgesetzt sind, ist jedoch eine ganz andere Frage, die hier abschlägig beantwortet wurde.

Die gewählte Mechanik, den Anreiz durch Verdoppelung der Zuzahlungsgrenze bei Eintritt einer Krebserkrankung zu schaffen, ist problematisch, weil sie nicht von Erkenntnissen der Verhaltensökonomik profitiert, die für einen zeitnäheren Anreiz sprechen würden; weil der als Anreiz fungierende Nachteil der höheren Zuzahlung sich zu einem Zeitpunkt manifestiert, in dem sich Patienten in einer schwierigen und verletzlichen Situation befinden; weil die Möglichkeit, dass Patienten Ärzte bitten, in ihrem Interesse der GKVen falsche Mitteilungen zu machen, nach der hier durchgeführten empirischen Forschung plausibel ist und das ArztPatienten-Verhältnis belastet (vgl.: Bundesärztekammer 2007); weil eine Gruppe von Chronikern gegenüber allen anderen ungleich behandelt wird; und

\section{Es wäre sinnvoller, das} Erfordernis abzuschaffen, eine angeratene Behandlung niemals abzulehnen, und alleine die Beratungs-Regelung umzusetzen.

weil trotz des intrusiven Charakters der Regelung in der Rechtfertigung keine klare Evidenz zum Gesundheitsverbesserungs- und Kosteneinsparungspotenzial im Vergleich zu anderen Ansätzen bereitgestellt wurde.

Es wäre daher sinnvoller, das Erfordernis abzuschaffen, eine angeratene Behandlung niemals abzulehnen, selbst wenn dieser zuvor zugestimmt wurde, und allein die Beratungs-Regelung umzusetzen. Für diese sollte eine explizite und systematische Kostenmodellierung vorgenommen werden, um zu ermitteln, ob nicht ein zeitnäherer Anreiz durch Gewinnbeteiligung statt durch Kostenverlagerung geschaffen werden kann (analog zu \65a SGB V). Insbesondere angesichts der ad hoc-Annahmen des Nationalen Krebsplans, denen zufolge ein dramatischer Anstieg der Kosten der medikamentösen Krebstherapie bevorsteht, sollte hier ein Potenzial vorhanden sein.

In jedem Fall sollte parallel dazu untersucht werden, mit welchen anderen
Mitteln sich die Screening-Teilnahme erhöhen lässt (es wurde auf die etwa dreifach höheren Raten in den USA hingewiesen). Ferner ist es erforderlich, Versicherte direkt über die Regelung zu informieren und sicherzustellen, dass die Beratung nicht-direktiv erfolgt: diese beiden Aspekte sind von besonderer Wichtigkeit, falls keine weiteren Änderungen zur gegenwärtigen Chronikerrichtline durchsetzbar sein sollten. In der Evaluation sollte insbesondere auch die Auswirkung der Regelung auf Populationen mit unterschiedlichem Sozialstatus und unterschiedlicher Gesundheitsmündigkeit berücksichtigt werden.

Die Diskussion hat hoffentlich auch gezeigt, welchen Beitrag der hier vorgeschlagene Rahmen zur Analyse von Regelungen zur Förderung der Eigenverantwortung leisten kann (und welchen nicht, vgl. Rid 2009): während er

keine Letztbegründung konkreter Regelungen über Rückbindung an eine umfassende normative Theorie leisten kann (die dann jedoch gegen andere umfassende Theorien verteidigt werden müsste), zeigte sich in der Auffächerung der sieben analytischen Gebiete hoffentlich dennoch, dass dieses Verfahren helfen kann, die wesentlichen Elemente fokussiert $\mathrm{zu}$ betrachten, und auf diese Weise Fortschritte dabei zu erzielen, wie angemessene (und weniger angemessene) Begründungen zu unterscheiden sind. 


\section{Literatur}

American Cancer Society. 2011. Colorectal Cancer Facts and Figures, 2011-2013. Atlanta, Georgia: American Cancer Society.

Brenner, Hermann, Lutz; Altenhofen, and Michael Hoffmeister. 2010. Zwischenbilanz der Früherkennungskoloskopie nach acht Jahren - Eine Hochrechnung und erste Ergebnisse. Dtsch Arztebl Int 43 (43): 753-9.

Bundesärztekammer. 2007. Beschlussprotokoll des 110. Deutschen Ärztetages vom 15-18. Mai in Münster. Berlin: Bundesärztekammer.

Bundesministerium für Gesundheit. 2007. BMG-Pressemitteilung Nr. 113 vom 17.12.2007Medizinische Vorsorge wird gestärkt.

\section{Bundesministerium für Gesundheit. 2008.}

Bekanntmachung eines Beschlusses des Gemeinsamen Bundesausschusses über eine Änderung der Chroniker-Richtlinie: Feststellung therapiegerechten Verhaltens. BAnz. Nr. 47 47: 1094.

Bundesministerium für Gesundheit. 2011. Nationaler Krebsplan, Handlungsfeld 1: Ziele-Papier 2 b Weiterentwicklung der Darmkrebsfrüherkennung. Bonn

Bundesregierung. 2006. Entwurf eines Gesetzes zur Stärkung des Wettbewerbs in der Gesetzlichen Krankenversicherung (GKVWettbewerbsstärkungsgesetz - GKV-WSG), 25 Oktober 2006.

Buyx, A. M. 2005. Eigenverantwortung Verteilungskriterium im Gesundheitswesen? Dtsch Med Wochenschr 130 (24): 1512-5

Cappelen, AW, and OF Norheim. 2005. Responsibility in health care: a liberal egalitarian approach. J Med Ethics 31 (8): 476-480.

Daniels, N. 2000. Accountability for reasonab leness. BMJ 321 (7272): 1300-1301.

Daniels, N. 2007. Just Health: Meeting Health Needs Fairly. Cambridge: CUP.

Daniels, N., und J. Sabin. 1999. Decisions about access to health care and accountability for reasonableness. Journal of Urban Health 76 (2): 176-191.

Deutscher Bundestag. 2007. Bundestagsplenarprotokoll 16/196, Stenografischer Bericht, 80. Sitzung, Berlin, Freitag, den 2. Februar 2007.
Frederick, S.; Loewenstein, G.; O'Donoghue, T. 2002. Time Discounting and Time Preference: A Critical Review. Journal of Economic Literature 40 (2): 351-401

Gemeinsamer Bundesausschuss. 2007. Beschluss des Gemeinsamen Bundesausschuss über eine Änderung des Chroniker-Richtlinie: Feststellung therapiegerechten Verhaltens. Siegburg: G-BA.

Huster, S. 2012. Solidarität und Eigenverantwortung - Spannung oder Gleichklang? Z. Evid. Fortbild. Qual. Gesundh. wesen [im Druck].

Höfling, W. 2009. Recht auf Selbstbestimmung versus Pflicht zur Gesundheit. Z. Evid Fortbild. Oual. Gesundh. wesen 103: 286-292.

Koch, K., Scheibler, F. 2007. Einstellungen und Informationsstand zur Früherkennung: Informiert und doch getäuscht? In: Böcken, J., Braun, B., Amhof, R.: Gesundheitsmonitor 2007 Verlag Bertelsmannstiftung.

Le Grand, J. 1991. Equity and choice. An essay in economics and applied philosophy. London: Harper Collins Academic.

Loewenstein, G, T Brennan, and K Volpp. 2007. Asymmetric paternalism to improve health behaviors. JAMA 298: 2415-2417.

Marckmann, Georg. 2005. Eigenverantwortung als Rechtfertigungsgrund für ungleiche Leistungsansprüche in der Gesundheitsversorgung? In Gleichheit und Gerechtigkeit in der modernen Medizin, edited by O. Rauprich, Marckmann, Georg, Vollmann, Jochen. Paderborn: Mentis.

Mühlhauser, Ingrid. 2007. Früherkennung und Prävention: Ist Vorbeugen besser als Heilen? Dtsch Arztebl 104 (25): A-1804 / B-1589 / C-1529.

Mühlhauser, Ingrid 2011. Vollständige und verständliche Daten sind nötig [Complete and Comprehensible Data Are Required]. Dtsch Arztebl Int 108 (10): 171

Rid, A. 2009. Justice and procedure: how does "accountability for reasonableness" result in fair limit-setting decisions? Journal of Medical Ethics 35 (1): 12-16.

Robert Koch-Institut. 2010. Krebs in Deutsch land 2005/2006. Häufigkeiten und Trends. 7 . Ausgabe. Berlin.
Schmidt, H. 2007a. Personal responsibility for health-developments under the German Healthcare Reform 2007. Eur J Health Law 14 (3): 241-50.

Schmidt, H. 2007b. Patients' charters and health responsibilities. BMJ 335 (7631): $1187-1189$

Schmidt, H. 2008. Bonuses as incentives and rewards for health responsibility: a good thing? J Med Philos 33 (3): 198-220.

Schmidt, H. 2008. Bonuses as Incentives and Rewards for Health Responsibility: A Good Thing? 10.1093/jmp/jhnoo7. J Med Philos 33 (3): 198-220.

Schmidt, H. 2009. Just health responsibility. J Med Ethics 35 (1): 21-6.

Schmidt, H. 2012. Anreize für Eigenverantwor tung: Begriffsbestimmung und Evidenzlage. Z. Evid. Fortbild. Qual. Gesundh. wesen [im Druck].

Sieg, A., and H. Brenner. 2007. Cost-saving analysis of screening colonoscopy in Germany. Z Gastroenterol 45 (9): 945-51.

von dem Knesebeck, O., and A. Mielck. 2009. Soziale Ungleichheit und gesundheitliche Versorgung im höheren Lebensalter. Z. Gerontol. Geriatr. 42 (1): 39-46

Winawer, S. J., A. G. Zauber, M. N. Ho, M. J. O’Brien, L. S. Gottlieb, S. S. Sternberg, J. D. Waye, M. Schapiro, J. H. Bond, J. F. Panish, and et al. 1993. Prevention of colorectal cancer by colonoscopic polypectomy. The National Polyp Study Workgroup. N Engl J Med 329 (27) 1977-81.

\section{Wuppermann, D, I Wuppermann, and JF}

Riemann. 2009. Aktueller Wissensstand der Bevölkerung zur Darmkrebsvorsorge - eine Untersuchung der Stiftung LebensBlicke mit dem Institut für Demoskopie in Allensbach Z Gastroenterol 47: 1132-6. 